\title{
3 Research S Suare \\ Does Concomitant DCIS Affect the Clinical Outcome in Breast Cancer Patients with Invasive Ductal Carcinoma: an Asian Perspective
}

Wai Peng Lee ( $\nabla$ waipenglee83@gmail.com )

Changi General Hospital Department of General Surgery https://orcid.org/0000-0001-6997-1524

Spoorthi Shetty Sudhakar

Changi General Hospital Department of General Surgery

Chin Mui Jaime Seah

Changi General Hospital Department of General Surgery

Pei Ting Tan

Changi General Hospital

Su Ming Tan

Changi General Hospital Department of General Surgery

\section{Research Article}

Keywords: Ductal carcinoma in situ (DCIS), invasive ductal carcinoma (IDC)

Posted Date: April 26th, 2021

DOl: https://doi.org/10.21203/rs.3.rs-452411/v1

License: (c) (i) This work is licensed under a Creative Commons Attribution 4.0 International License.

Read Full License

Version of Record: A version of this preprint was published at European Journal of Cancer on October 1st, 2020. See the published version at https://doi.org/10.1016/S0959-8049(20)30788-7. 


\section{Abstract}

\section{Background}

Ductal carcinoma in situ (DCIS) is an established precursor to invasive ductal carcinoma (IDC) and its coexistence with IDC appear to favour reduced biological aggressiveness. Its prognostic implication and ability to affect clinical outcome has been understudied in Asia. This study aims to explore if concomitant DCIS affects the clinical behavior and outcomes among Asians.

\section{Methods}

Stage I to III breast cancer patients with histologically proven invasive ductal carcinoma, diagnosed and treated in a single institution from 1 June 2004 to 30 June 2014 were included in this study. Statistical analyses were conducted using $\mathrm{x}^{2}$ test, independent $\mathrm{T}$ - test, multi-variate logistic regression and KaplanMeier test.

\section{Results}

A total of 818 patients were identified, including 224 and 594 patients with isolated IDC (No-DCIS) and IDC with coexisting DCIS (IDC-DCIS) respectively. Patients with IDC-DCIS were found to have smaller tumours (median: $22 \mathrm{~mm}, p<=0.01)$, estrogen receptor positivity $(p=0.001)$, progesterone receptor positivity $(p<0.001)$ and associated with better pathological stage $(p=0.001)$. Patients with No-DCIS were 1.6 times more likely to develop disease progression ( $95 \% \mathrm{Cl}: 1.1-2.3, \mathrm{p}=0.027)$ and subsequently associated with distant recurrences $(20.5 \%$ vs $13.6 \%, p=0.02)$. The breast cancer specific 5 year overall survival rate for patients with No-DCIS and those with IDC-DCIS was $90.9 \%$ (95\% Cl: $86.2 \%-94.5 \%$ ) and 93.7\% (95\% Cl: 91.4- 95.5\%) respectively $(p=0.202)$.

\section{Conclusion}

The presence of DCIS component in IDC among Asians is associated with favourable tumour biological profile, thereby indicating reduced disease aggressiveness. Our study is the first to report the clinical significance in terms of disease progression and distant recurrences among Asians.

\section{Trial registration}

This study had received the approval of the institutional ethics committee prior to its commencement (IRB Ref No: 2019/2884).

\section{Background}

Screening mammography has led to a rising detection of early breast cancer, namely ductal carcinoma in situ (DCIS). Though the assumption that invasive cancers are likely to derive from pre-existing $\mathrm{DCIS}^{1,2}$, IDC may still evolve de novo in up to $21.4 \%$ of cases worldwide. A probable delay in transformation from 
insitu to invasive form is belived to account for tumours with coexisting DCIS demonstrating lesser biological aggresiveness ${ }^{3,4}$. Recent studies have linked tumours with IDC-DCIS with better clinical features such as smaller and lower grade tumours and lower probability of lymph node invasion. Nonetheless, it remains controversial if clinical outcomes such as recurrences and overall survival rates are affected ${ }^{5,6}$. The clinical impact of concomitant DCIS in invasive cancers involving Asians has been understudied. Hence, our study aims to address if coexisting DCIS affects the tumour characteristics and clinical outcome such as recurrences, disease progression and overall survival among Asians.

\section{Methods}

A retrospective analysis was performed on our prospectively collected breast cancer database with an inclusion period from 1 June 2004 to 30 June 2014. This database comprises of patients who were diagnosed and underwent treatment in a specialized breast unit of a single institution. Only patients with definitive histo-pathology diagnosis for invasive ductal carcinoma were selected for evaluation. Patients diagnosed with metastatic disease were excluded. Patients with bilateral breast cancer were included as two separate study cases. Figure 1 shows the inclusion and exclusion criteria that resulted in our main study cohort of 818 patients.

Data collected include clinical characteristics, histopathological information and the type of surgery performed. The TNM classification was based on the latest edition of the American Joint Committee on Cancer at the time of reporting of the histopathology specimen.

Sentinel lymph node biopsy (SLNB) was performed in all patients with a preoperative diagnosis of infiltrative ductal carcinoma and in the absence of preoperative clinical or radiological evidence of nodal involvement. Axillary lymphadenectomy was performed in patients who had preoperative diagnosis of nodal tumour or macrometastasis on frozen section. Tumour histopathology and the number of lymph nodes involved were evaluated by routine hematoxylin- eosin (H\& E) staining.

The cases were divided into two groups: invasive ductal carcinoma (No-DCIS) and invasive ductal carcinoma with ductal carcinoma in situ (IDC-DCIS).

This study had received the approval of the institutional ethics committee (IRB Ref No: 2019/2884).

Quantitative data are shown as median or mean of their values and their variability is expressed as range or standard deviation (SD), as specified for each analysis. Qualitative values are shown as absolute values or percentages. Categorical data was presented in frequency and percentage and association between subjects' characteristics and DCIS were tested using Chi-square test.

Numerical data was presented in mean (standard deviation) and association was tested using Independent T-test if normal distribution was fulfilled. Otherwise, data was presented in median and interquartile rage (IQR) and Mann-Whitney $U$ test was performed to test for the association between subjects' 
characteristics and DCIS status. Logistic regression was performed to identify the risk of developing disease progression between DCIS and non-DCIS patients.

Survival analysis and duration to disease free progression between DCIS and non-DCIS were performed using Kaplan-Meier with log rank test to assess for statistical differences.

Propensity scores were calculated using a multiple Logistic Regression with clinical relevant variables: age, overall tumor stage, menopausal status, hormonal status and cerB2 receptor status. Nearestneighbor matching with maximum difference of $5 \%$ approach in 1:1 fashion was performed to match the propensity scores values of the DCIS and non-DCIS group.

Statistical significance was set at $\mathrm{P}<0.05$. Analysis was performed using SPSS version 21 (IBM Corp., Armonk, USA).

\section{Results}

A total of 818 cases from 1 June 2004 to 30 June 2014 were included. No-DCIS was present in 224 (27.4\%) cases while 594 (72.6\%) had IDC-DCIS. The mean age at diagnosis was 55.6 years (42.1-69.1). The mean follow up period in our study was 93.7 months (47.2-140.2). The ethnic distribution of our study cohort was consistent with our national demographics. The clinicopathological features and the analysis between the two groups are shown in Table 1. Tumors were either T1 (43.8\%) or T2 (46.3\%). Five hundred and one patients (61.2\%) had no nodal involvement (N0). Patients were found to have Stage 1 $(32.6 \%)$ and $2(53.2 \%)$ cancer, followed by Stage 3 (14.2\%). We excluded patients with stage 4.

82.9\% had presented with a clinically palpable breast lump. 13.1\% were screen detected and hence, asymptomatic (Supplementary Table 1). Others had nipple discharge, breast pain or skin changes and were subsequently evaluated to have cancer. Nine patients presented with bilateral tumours, either synchronous or metachronous.

Lumpectomy was performed in 170 patients while 645 patients had mastectomy as a form of surgical treatment for their condition. One hundred and seventy four patients $(78.0 \%)$ in the No-DCIS group and $471(79.6 \%)$ in the IDC-DCIS group underwent mastectomy $(p=0.630)$.

Though majority of tumours in both groups were found to be grade $3(48.2 \%)$, this result was not statistically significant $(p=0.171)$. Similarly, there was no statistical significance upon comparison of the presence of lymphovascular invasion between both groups $(p=0.211)$.

The median size of the invasive tumour of the No-DCIS group was $27 \mathrm{~mm}$ while the IDC-DCIS group was $22 \mathrm{~mm}(p<0.001)$. Patients with IDC-DCIS were associated with lower T stage $(p<0.001)$ and better overall pathological stage $(p=0.001)$. Furthermore, patients with IDC-DCIS were likely to express positivity in hormonal receptors (estrogen receptor, $p=0.001$; progesterone receptor, $p<0.001$ ) and cer $B 2$ receptor $(p<0.001)$. 
The mean duration of follow up was 93.7 months. Clinical outcomes have been summarised in Table 2 . One hundred and fifteen distant recurrences were noted, with No-DCIS group having $42(20.5 \%)$ and the IDC-DCIS group having $73(13.6 \%)(p=0.020)$. Forty eight local recurrences were recorded; No-DCIS group had $17(8.1 \%)$ while IDC-DCIS group had $31(5.8 \%)(p=0.72)$.

Patients with No-DCIS were 1.6 times more likely to develop disease progression in contrast to IDC-DCIS (OR: 1.6, 95\% Cl: 1.1-2.6, $p=0.027$ ). Patients with IDC-DCIS recorded a longer survival (14.2 years vs 13.9 years). Log rank test showed that this was not statistically significant $(p=0.274)$. Similarly, patients with IDC-DCIS had an average 12.7 years of disease free progression as compared to those with No-DCIS $(12.5$ years $)(p=0.600)$.

The breast cancer specific 5 year overall survival rate was performed in 163 pairs matched patients with the aid of propensity score matching (PSM) (Fig. 2)and was $92.02 \%$ (95\% Cl 90.9\% - 94.7\%). Cox proportion hazard ratio showed that patients with IDC-DCIS more likely to progress faster to death (Table 3). Adjustment by age, overall tumour stage, menopausal status, hormonal receptor and cerB2 status showed no significant difference in the 5 year overall survival rate $(p=0.608)($ Table 3$)$. 
Table 1

Clinicopathologic features of the entire study population and No-DCIS and IDC-DCIS study groups

\begin{tabular}{|c|c|c|c|c|}
\hline Variable & $\begin{array}{l}\text { Total }(n= \\
818)\end{array}$ & $\begin{array}{l}\text { No-DCIS }(n= \\
224)\end{array}$ & $\begin{array}{l}I D C-D C I S(n= \\
594)\end{array}$ & $p$ value \\
\hline Age mean years (SD) & $55.6(13.5)$ & $55.3(14.5)$ & $55.7(13.1)$ & $\begin{array}{l}P= \\
0.739\end{array}$ \\
\hline \multicolumn{5}{|l|}{ Age group } \\
\hline$<40$ & $82(10.0)$ & $31(14.5)$ & $51(8.6)$ & $\begin{array}{l}p= \\
0.826\end{array}$ \\
\hline $40-49$ & $224(27.3)$ & $50(23.3)$ & $174(29.3)$ & \\
\hline $50-59$ & $218(26.7)$ & $61(28.5)$ & $157(26.4)$ & \\
\hline $60-69$ & $147(18.0)$ & $41(19.2)$ & $106(17.8)$ & \\
\hline$>=70$ & $147(18.0)$ & $31(14.5)$ & $106(17.8)$ & \\
\hline \multicolumn{5}{|l|}{ Ethnicity } \\
\hline Chinese & $588(71.9)$ & $162(72.3)$ & $426(71.7)$ & $\begin{array}{l}P= \\
0.397\end{array}$ \\
\hline Malay & $135(16.5)$ & $42(18.8)$ & $93(15.7)$ & \\
\hline Indian & $46(5.6)$ & $9(4.0)$ & $37(6.2)$ & \\
\hline Others & $49(6.0)$ & $11(4.9)$ & $38(6.4)$ & \\
\hline Follow-up duration & $93.7(46.5)$ & $91.6(49.3)$ & $93.8(45.5)$ & $\begin{array}{l}P= \\
0.541\end{array}$ \\
\hline Invasive tumour size, median (mm) & $24(15-35)$ & $27(18-45)$ & $22(14-31.3)$ & $\begin{array}{l}P< \\
0.001\end{array}$ \\
\hline \multicolumn{5}{|l|}{ Tumour status n (\%) } \\
\hline 1 & $358(43.8)$ & $78(21.8)$ & $280(78.2)$ & $\begin{array}{l}P< \\
0.001\end{array}$ \\
\hline 2 & $379(46.3)$ & $106(28.0)$ & $273(72.0)$ & \\
\hline 3 & $80(9.8)$ & $40(50.0)$ & $40(50.0)$ & \\
\hline \multicolumn{5}{|l|}{ Nodal status n (\%) } \\
\hline 0 & $501(61.2)$ & $130(25.9)$ & $371(74.1)$ & $\begin{array}{l}P= \\
0.752\end{array}$ \\
\hline 1 & $211(25.8)$ & $59(28.0)$ & $152(72.0)$ & \\
\hline 2 & $93(11.4)$ & $28(30.1)$ & $65(69.9)$ & \\
\hline
\end{tabular}




\begin{tabular}{|c|c|c|c|c|}
\hline Variable & $\begin{array}{l}\text { Total }(n= \\
818)\end{array}$ & $\begin{array}{l}\text { No-DCIS }(n= \\
224)\end{array}$ & $\begin{array}{l}I D C-D C I S(n= \\
594)\end{array}$ & $p$ value \\
\hline 3 & $1(0.1)$ & $0(0.0)$ & $1(100.0)$ & \\
\hline \multicolumn{5}{|c|}{ Overall Stage n (\%) } \\
\hline 1 & $267(32.6)$ & $55(20.6)$ & $212(79.4)$ & $\begin{array}{l}P= \\
0.001\end{array}$ \\
\hline 2 & $435(53.2)$ & $125(28.7)$ & $310(71.3)$ & \\
\hline 3 & $116(14.2)$ & $44(37.9)$ & $72(62.1)$ & \\
\hline \multicolumn{5}{|c|}{ Tumour grade $\mathrm{n}(\%)$} \\
\hline 1 & $126(15.4)$ & $30(23.8)$ & $96(76.2)$ & $\begin{array}{l}P= \\
0.171\end{array}$ \\
\hline 2 & $272(33.3)$ & $62(22.8)$ & $210(77.2)$ & \\
\hline 3 & $394(48.2)$ & $114(28.9)$ & $280(71.1)$ & \\
\hline \multicolumn{5}{|c|}{$\begin{array}{l}\text { Presence of lymphovascular } \\
\text { invasion } n(\%)\end{array}$} \\
\hline Yes & $336(43.4)$ & $80(39.6)$ & $256(44.7)$ & $\begin{array}{l}P= \\
0.211\end{array}$ \\
\hline No & $439(56.6)$ & $122(60.4)$ & $317(55.3)$ & \\
\hline \multicolumn{5}{|c|}{ Estrogen receptor n (\%) } \\
\hline Positive & $579(70.8)$ & $135(23.3)$ & $444(76.7)$ & $\begin{array}{l}P= \\
0.001\end{array}$ \\
\hline Negative & $230(28.1)$ & $81(35.2)$ & $149(64.8)$ & \\
\hline \multicolumn{5}{|c|}{ Progesterone receptor n (\%) } \\
\hline Positive & $494(60.4)$ & $110(22.3)$ & $384(77.7)$ & $\begin{array}{l}P< \\
0.001\end{array}$ \\
\hline Negative & $315(38.5)$ & $106(33.7)$ & $209(66.3)$ & \\
\hline \multicolumn{5}{|c|}{ Her-2 receptor $n(\%)$} \\
\hline Positive & $203(24.8)$ & $28(13.8)$ & $175(86.2)$ & $\begin{array}{l}P< \\
0.001\end{array}$ \\
\hline Negative & $562(68.7)$ & $179(31.9)$ & $383(68.1)$ & \\
\hline \multicolumn{5}{|c|}{ Menopausal status n (\%) } \\
\hline Pre-menopausal & $431(53.9)$ & $119(54.1)$ & $268(46.2)$ & $\begin{array}{l}P= \\
0.940\end{array}$ \\
\hline
\end{tabular}




\begin{tabular}{|lllll|}
\hline Variable & Total $(\boldsymbol{n}=$ & $\begin{array}{l}\text { No-DCIS }(\boldsymbol{n}= \\
\text { 224) }\end{array}$ & $\begin{array}{l}\text { IDC -DCIS }(\boldsymbol{n}= \\
\text { 594) }\end{array}$ & p value \\
\hline Post-menopausal & $369(46.1)$ & $101(45.9)$ & $312(53.8)$ & \\
\hline Surgical type $\mathrm{n}(\%)$ & & & & \\
\hline Conservation & $170(26.4)$ & $49(22.0)$ & $121(20.4)$ & $\begin{array}{l}\mathrm{P}= \\
\text { Mastectomy }\end{array}$ \\
\hline
\end{tabular}

Table 2

Recurrences of the entire study population and No-DCIS and IDC-DCIS study groups

\begin{tabular}{|lllll|}
\hline Variable & Total $(\boldsymbol{n = 8 1 8})$ & No-DCIS $(\boldsymbol{n}=\mathbf{2 2 4})$ & IDC-DCIS $(\boldsymbol{n}=594)$ & $\boldsymbol{p}$ value \\
\hline Local recurrence $\mathrm{n}(\%)$ & & & & \\
\hline Yes & $48(6.4)$ & $17(8.1)$ & $31(5.8)$ & $\mathrm{p}=0.746$ \\
\hline No & $698(93.6)$ & $192(91.9)$ & $506(94.2)$ & \\
\hline Distant recurrence $\mathrm{n}(\%)$ & & & & \\
\hline Yes & $115(15.5)$ & $42(20.5)$ & $73(13.6)$ & $\mathbf{p}=0.020$ \\
\hline No & $627(84.5)$ & $163(79.5)$ & $464(86.4)$ & \\
\hline
\end{tabular}

Table 3

Utilization of Cox Proportion Hazard Ratio (Adjustment for age, overall tumour stage, menopausal status, hormonal receptor and cerB2 status)

\begin{tabular}{|c|c|c|c|c|}
\hline & Breast cancer related deaths (\%) & Mean, years $(95 \% \mathrm{Cl})$ & $H R(95 \%$ Cl) & $p$ value \\
\hline IDC-DCIS & 25/163 (15.3) & $13.30(12.68,13.92)$ & REF & \\
\hline No-DCIS & $21 / 163(12.9)$ & $13.56(12.96,14.17)$ & $0.86(0.48,1.54)$ & 0.608 \\
\hline
\end{tabular}

\section{Discussion}

Preliminary biological studies are able to demonstrate a biological difference between cancers with NoDCIS and those with IDC-DCIS. The current hypothesis for the presence of concomitant in situ disease in such cancers is that tumours do undergo cellular differentiation to transform into an invasive phenotype. This in turn led to the conclusion that this slow progression to the invasive component may then translate into a more favourable clinical prognosis 7,8 . Yet, only a handful of Western studies were able to recognize and associate this to possibly better prognostic features and clinical outcomes. Most yielded insignificant and inconsistent results ${ }^{9,10}$. The clinical significance and role of DCIS among the Asian population remains understudied. 
In Singapore, screening mammography is highly subsidized, making it extremely affordable for asymptomatic women. With the increasing awareness for screening mammography, detection of early cancers has also been on the rise. However, currently, the presence of DCIS in invasive cancers has no impact on systemic treatment, which depends solely on the pathological characteristics of the invasive component ${ }^{11}$.

There has yet to be a common consensus between the clinical features and prognostic implications for tumours with concomitant DCIS. With progressive published data among the Westerns, it is widely accepted that patients with IDC-DCIS may present at a younger age with smaller sized tumours with few or no lymph nodal involvement ${ }^{9,10}$. Our results indicated that IDC-DCIS is associated with smaller sized tumours and a lower overall clinical stage, thereby concurring with the hypothesis that tumours with concomitant DCIS were less biologically aggressive.

Likewise, some studies have showed that tumours with concomitant DCIS are likely to express ER, PR and cerB2 positivity as compared to tumours with No-DCIS ${ }^{13-15}$. Others disagree ${ }^{12,15}$. Our results showed that IDC-DCIS subjects were likely to express positivity in ER, PR and cerB2 receptors and this was statistically significant. While our data suggest that IDC-DCIS cancers may imply a less aggressive phenotype for patients with hormonal receptor or Her2 receptor positive cancers, we do acknowledge that triple negative malignancies may exhibit different biological behavior.

Researchers have since supported the preliminary theory of this slow evolution in deriving the invasive component and thereby resulting in a possibly better clinical prognosis. This study did demonstrate that the group with No-DCIS had higher percentage of distant recurrences compared to the group with IDCDCIS $(20.6 \%$ vs $13.5 \%, p=0.020)$. However, we were unable to draw a similar conclusion for local recurrence. This might be attributed to the small total number of patients in our cohort who had developed local recurrences over the surveillance period as a result of better compliance to local radiation therapy prescribed. Our study results also suggest that patients with No-DCIS were 1.6 times more likely to develop disease progression as compared to those with IDC-DCIS, in spite of the adjuvant treatment given. These results add weight to the current speculation that the absence of coexisting DCIS is associated with poorer prognostic features and outcomes among Asian patients, especially in terms of distant recurrences and disease progression. Unfortunately, due to our small sample size of breast cancer related deaths, we were unable to detect a significant difference in the breast cancer specific 5 year overall survival among the 2 groups.

The breast cancer specific 5 year overall survival rate was analysed with PSM. No significant results were found between the group with No-DCIS and IDC-DCIS $(92.02 \%, 95 \% \mathrm{Cl}=90.9 \%-94.7 \%, \mathrm{p}=0.608)$. PSM is regarded as an advanced statistical technique to minimize any possible confounders in an observational study. It serves to reduce possible treatment assignment bias and mimic randomization ${ }^{16}$. We utilised PSM to assess if concomitant DCIS affects the 5 year breast cancer overall survival rate after adjusting for certain covariates as mentioned above but we were unable demonstrate a more favourable breast 
cancer specific 5 year overall survival rate. It might be attributed to the fact that both genomic profiles are highly similar ${ }^{17,18}$.

Lastly, we do recognize the limitations of this study. Being a retrospective analysis, any incomplete data namely, less detailed histology reports in the early years of the 21 st century, had to be excluded. Other information such as patients' details may have been missing during the early days of data entry. Other inherent biases associated with retrospective study have to be considered. Secondly, analysis of tumour specimens had been performed in the absence of central pathologic review, hence establishment of details such as presence of isolated tumour cells (ITC) infiltration and micrometastases (mic) in lymph nodes were absent. Thirdly, our sample size was too small to demonstrate a significant difference in the 5 year breast cancer specific survival. Unlike the Western studies, the clinical outcomes among Asians such as recurrences and disease free outcomes as a result from tumours with coexisting DCIS has been understudied $^{19,20}$. This study, with a long median follow up of 94 months, is to add weight to current findings and further strengthen the belief that coexisting DCIS does lead to better tumour profile. We are the first to document the clinical significance of concomitant DCIS in the presence of invasive cancers in terms of disease progression and distant recurrences.

\section{Conclusion}

With the limited data among Asian population, our study remains the first to demonstrate improved clinical outcomes in terms of disease progression and distant recurrences. Henceforth, this allows clinicians to better prognosticate and consider vigilant clinical surveillance of patients diagnosed with isolated IDC in remission.

\section{Declarations}

\section{Ethics approval and consent to participate}

This study had received the approval of the institutional ethics committee prior to its commencement (IRB Ref No: 2019/2884). A waiver of consent was permitted.

\section{Consent for publication}

Not applicable

\section{Availability of data and materials}

The datasets used during the current study are available from the corresponding author on reasonable request.

\section{Competing interests}

The authors declare that they have no competing interests. 


\section{Funding}

This study did not receive any funding.

\section{Authors' contributions}

LWP interpreted the data set and was a major contributor in writing the manuscript. SSS and SCM were heavily involved in data collection and data entry. TPT performed the analysis and interpreted the data set. TSM had edited the manuscript prior to submission. All authors read and approved the final manuscript

\section{Acknowledgements}

Not applicable

\section{References}

1. Burstein HJ, Polyak K, Wong, et al. Ductal carcinoma in situ of the breast. New England journal of medicine. 2004;350:1430-41.

2. Sgroi DC. Preinvasive breast cancer. Annu Rev Pathol. 2010;5:193-221.

3. Dzierzanowski M, Melville KA, Barnes PJ, et al. Ductal carcinoma in situ in core biopsies containing invasive breast cancer: correlation with extensive intraductal component and lumpectomy margins. $J$ Surg Oncol. 2005;90(2):71-6.

4. Logullo AF, Godoy AB, Mourão-Neto M, et al. Presence of ductal carcinoma in situ confers an improved prognosis for patients with T1NOMO invasive breast carcinoma. Braz J Med Biol Res. 2002;35(8):913-9.

5. Dieterich $\mathrm{M}$, et al. Accompanying DCIS in breast cancer patients with invasive ductal carcinoma is predictive of improved local recurrence-free survival. Breast. 2014;23:346-51.

6. Carabias-Meseguer P, et al. Influence of the in situ component in 389 infiltrating ductal breast carcinomas. Breast Cancer. 2013;20:213-7.

7. Wong H, Lau S, Yau T, et al. Presence of an in situ component is associated with reduced biological aggressiveness of size-matched invasive breast cancer. Br JCanc. 2010;102:1391-6.

8. Chagpar AB, McMasters KM, Sahoo S, et al. Does ductal carcinoma in situ accompanying invasive carcinoma affect prognosis? Surgery. 2009;146:561-7.

9. Sebastian Sebastian C, Garcia Mur C, Cruz Ciria S, et al. Imaging and histologic prognostic factors in triple-negative breast cancer and carcinoma in situ as a prognostic factor. Radiologia. 2016;58:28393.

10. Goh CW, Wu J, Ding S, et al. Invasive ductal carcinoma with coexisting ductal carcinoma in situ (IDC/DCIS) versus pure invasive ductal carcinoma (IDC): a comparison of clinicopathological 
characteristics, molecular subtypes, and clinical outcomes. J Cancer Res Clin Oncol. 2019;145:1877-86.

11. Gradishar WJ, et al. Invasive Breast Cancer Version 1.2016, NCCN Clinical Practice Guidelines in Oncology. J Natl Compr Canc Netw. 2016;14:324-54.

12. Dieterich $M$, Hartwig F, Stubert J, et al. Accompanying DCIS in breast cancer patients with invasive ductal carcinoma is predictive of improved local recurrence-free survival. Breast. 2014;23(4):346-51.

13. Kim JY, Han W, Moon HG, et al. Grade of ductal carcinoma in situ accompanying infiltrating ductal carcinoma as an independent prognostic factor. Clin Breast Cancer. 2013;13(5):385-91.

14. Wong H, Lau S, Yau T, Cheung P, et al. Presence of an in situ component is associated with reduced biological aggressiveness of sizematched invasive breast cancer. Br J Cancer. 2010;102(9):1391-6.

15. Logullo AF, Godoy AB, Mourão-Neto M, et al. Presence of ductal carcinoma in situ confers an improved prognosis for patients with T1NOMO invasive breast carcinoma. Braz J Med Biol Res. 2002;35(8):913-9.

16. Brookhart MA, Schneeweiss S, Rothman KJ, et al. Variable selection for propensity score models. Am J Epidemiol. 2006;163(12):1149-56.

17. Schorr MC, Pedrini JL, Savaris RF, Zettler CG. Are the pure in situ breast ductal carcinomas and those associated with invasive carcinoma the same? Appl Immunohistochem Mol Morphol. 2010;18(1):51-4.

18. lakovlev VV, Arneson NC, Wong V, et al. Genomic differences between pure ductal carcinoma in situ of the breast and that associated with invasive disease: a calibrated study. Clin Cancer Res. 2008;14(14):4446-54.

19. $H$ Chen, $F$ Bai, $M$ Wang, $M$ Zhang, et al. The prognostic significance of co-existence ductal carcinoma in situ in invasive ductal breast cancer: a large population-based study and a matched case-control analysis. Ann Transl Med 2019 Sep; 7(18): 484

20. Goh CW, Wu J, Ding S, Lin C, et al. Invasive ductal carcinoma with coexisting ductal carcinoma in situ (IDC/DCIS) versus pure invasive ductal carcinoma (IDC): a comparison of clinicopathological characteristics, molecular subtypes, and clinical outcomes. J Cancer Res Clin Oncol. 2019 Jul;145(7):1877-86.

\section{Figures}




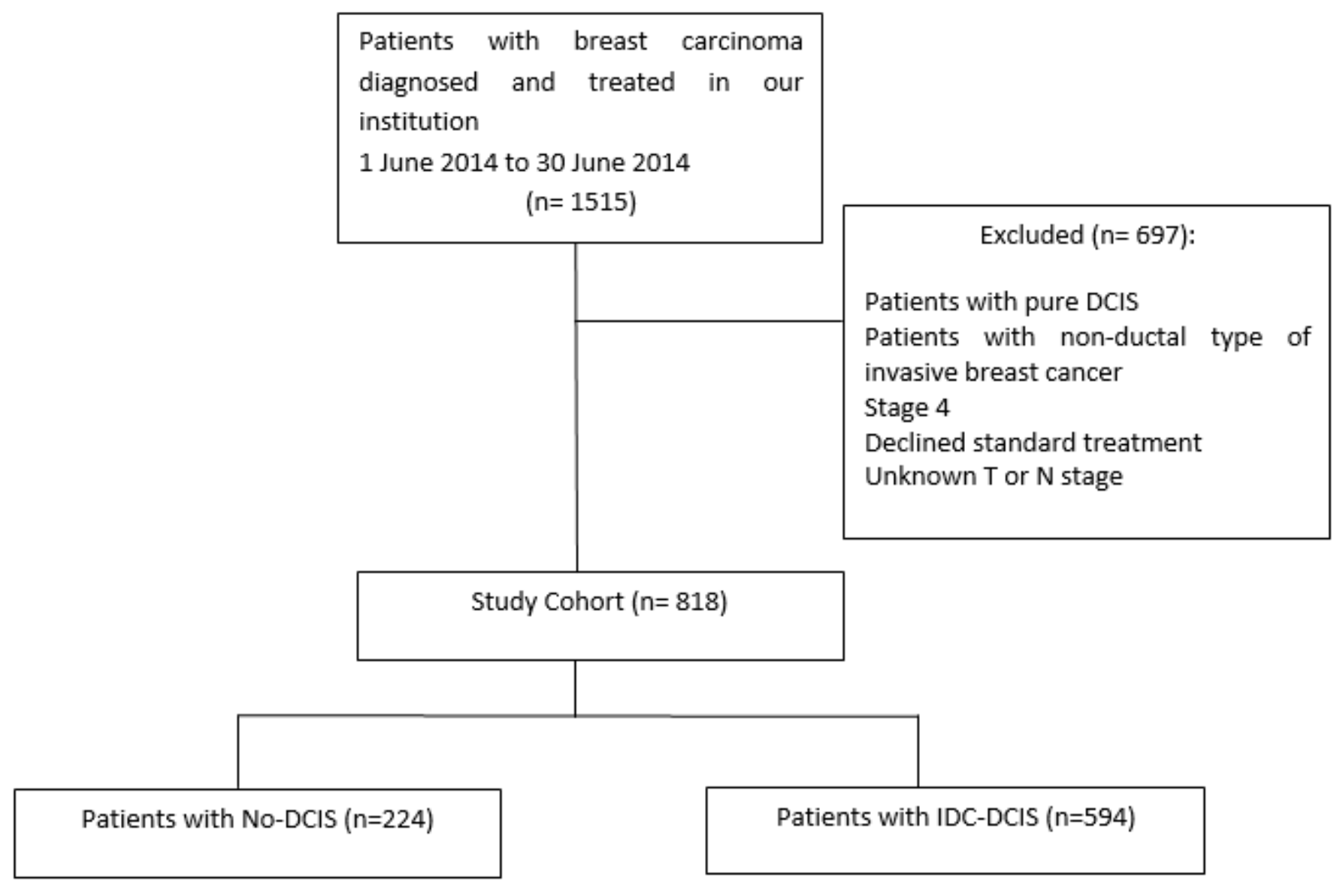

Figure 1

Study Cohort Flow Diagram.
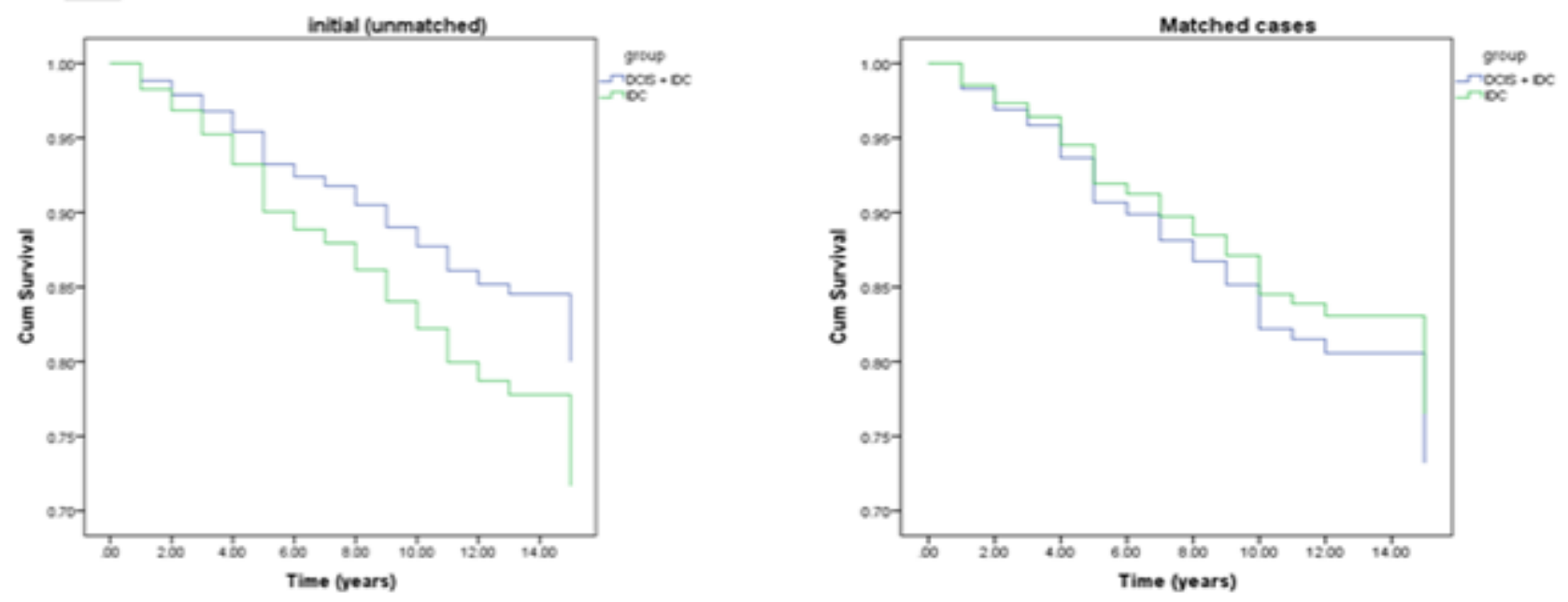

Figure 2

Breast Cancer Specific 5 year Overall Survival before (left) and after (right) PSM.

\section{Supplementary Files}


This is a list of supplementary files associated with this preprint. Click to download.

- supplementalfile.doc 